\title{
Functional Differentiation in the Human Auditory and Language Areas Revealed by a Dichotic Listening Task
}

\author{
Ryuichiro Hashimoto,* Fumitaka Homae,* Kyoichi Nakajima, $†$ \\ Yasushi Miyashita,†' and Kuniyoshi L. Sakai*,§,1 \\ *Department of Cognitive and Behavioral Science, Graduate School of Arts and Sciences, The University of Tokyo, 3-8-1 Komaba, \\ Meguro-ku, Tokyo 153-8902, J apan; †Department of Physiology, The University of Tokyo School of Medicine, 7-3-1 Hongo, Bunkyo-ku, \\ Tokyo 113-0033, J apan; łICORP and §CREST, J apan Science and Technology Corporation (J ST), Tokyo, J apan
}

Received February 29, 2000

The human auditory cortex plays a special role in speech recognition. It is therefore necessary to clarify the functional roles of individual auditory areas. We applied functional magnetic resonance imaging (fMRI) to examine cortical responses to speech sounds, which were presented under the dichotic and diotic (binaural) listening conditions. We found two different response patterns in multiple auditory areas and language-related areas. In the auditory cortex, the medial portion of the secondary auditory area (A2), as well as a part of the planum temporale (PT) and the superior temporal gyrus and sulcus (ST), showed greater responses under the dichotic condition than under the diotic condition. This dichotic selectivity may reflect acoustic differences and attention-related factors such as spatial attention and selective attention to targets. In contrast, other parts of the auditory cortex showed comparable responses to the dichotic and diotic conditions. We found similar functional differentiation in the inferior frontal (IF) cortex. These results suggest that multiple auditory and language areas may play a pivotal role in integrating the functional differentiation for speech recognition. 2000 Academic Press

Key Words: speech recognition; functional magnetic resonance imaging (fMRI); the auditory cortex.

\section{INTRODUCTION}

Sensory information processing in the cerebral cortex has been characterized as involving multiple areas, that are hierarchically organized and functionally specialized (Felleman and Van Essen, 1991). It is known that speech recognition critically depends on specialized cortical regions, including auditory areas as well as language areas (Geschwind, 1979). While cytoarchi-

\footnotetext{
${ }^{1}$ To whom correspondence and reprint requests should be addressed. E-mail: sakai@mind.c.u-tokyo.ac.jp.
}

tectonic studies have identified multiple auditory areas (Brodmann, 1909; von Economo and Horn, 1930; Galaburda and Sanides, 1980; Rivier and Clarke, 1997), roles of these multiple areas in auditory analysis per se have yet to be elucidated. Using recently developed imaging techniques, differential cortical responses to speech stimuli could reveal functional differentiation in the auditory cortex. Previous imaging studies have examined the effects of cognitive factors involved in auditory perception under various paradigms. However, consistent results have not been obtained as to whether auditory attention modulates responses in the auditory cortex. Some studies have reported attentionrelated enhancement of responses to speech and nonspeech stimuli in the auditory cortex (Woldorff et al., 1993; O'Leary et al., 1996; F ujiwara et al., 1998; J äncke et al., 1999; Alho et al., 1999). Moreover, there is a claim that this enhancement is more prominent in higher auditory areas than that in the primary auditory area (A1); attention-related modulation of activity occurs mainly in Brodmann's area (BA) 42 or 22 (Grady et al., 1997), and further, BA 22 is more susceptible to auditory selective attention than BA 42 (Pugh et al., 1996). In contrast, positron emission tomography (PET) studies have reported that attention-related factors do not increase responses in the auditory cortex (Zatorre et al., 1992, 1999; Frith and Friston, 1996; Tzourio et al., 1997).

This controversy may be not only due to the differences of paradigms among these studies, but due to attentional influences on the cortical activities which are manifested in only some restricted regions of the auditory cortex. The anatomical works have shown that the auditory cortex is not organized as a homogeneous area and that the structure of the auditory cortex is highly variable among individuals (Penhune et al., 1996; Westbury et al., 1999). Therefore it is necessary that the auditory areas are mapped on an individual brain. The group analyses with PET and functional magnetic resonance imaging ( $\mathrm{fMRI}$ ), as well as 
difficulty in the identification of multiple sources with magnetoencephal ogram (MEG), may lead to conflicting results of attentional enhancement. To examine the functional roles of multiple auditory areas in speech recognition, we used $\mathrm{fMRI}$ with an individual analysis approach and a dichotic listening task, which has been well established as a paradigm of studying auditory attention (Cherry, 1953; Treisman, 1969). A dichotic listening (DIC) condition requires subjects to recognize only one of different messages that are presented simultaneously to different ears. Thus it involves attention-related factors which are often necessitated in real-life events, such as spatial orientation and extraction of a relevant message from the mixture of irrelevant messages (cocktail party phenomenon). As a contrasting condition to the dichotic listening task, we used a diotic (binaural) listening (DIO) task, in which the same messages are presented to both ears. By examining the cortical responses under these two conditions with speech stimuli, as well as under a control (CON) condition with listening to nonspeech sounds, we aimed to functionally parcellate the auditory and language areas into two kinds of functional regions: the regions with responses that may reflect attention-related factors, identified by the direct comparison DIC vs DIO, and the other regions with responses to speech stimuli compared with nonspeech sounds, identified by the comparison (DIO + DIC) vs CON. A portion of this study has been reported previously in abstract form (Hashimoto et al., 1999).

\section{MATERIALS AND METHODS}

\section{Subjects}

Seven male native J apanese speakers (ages: 20-32) participated in the present study. All subjects showed right-handedness (laterality quotients: 81-100) by the Edinburgh inventory (Oldfield, 1971). The subject was in a supine position in the magnet with eyes closed in a dark room and the subject's head was immobilized with padding inside the radio-frequency coil. During the experiment, the subject wore earplugs surrounding the plastic tubes for sound delivery, and scanner noise was further attenuated by insulating padding on external ears. Informed consent from each subject was obtained after the nature and possible consequences of the studies were explained. Approval for these experiments was obtained from the institutional review board of the University of Tokyo (Graduate school of Arts and Sciences), and that of the University of Tokyo School of Medicine.

\section{Auditory Stimuli}

We presented two kinds of auditory stimuli: speech and nonspeech sounds. Each type of stimuli consisted of targets to be attended and nontargets to be neglected by the subjects. For speech sounds, continuous sentences of a story ("Snow White" in J apanese) were divided into phrases at natural break points: "Mirror,/ who is / themost / beautiful woman / in this country?" For each target, we made three corresponding nontargets by randomizing the order of syllables of the target: kamigayo, kayomiga, and gakayomi (nontargets, phonotactically acceptable nonsense syllables) for kagami yo (target, "Mirror," in J apanese). Prior to the experiment, we showed the subject a script of the whole story without any nontargets.

All speech sounds were digitized (16 bit; the normal audio cut-off, 11,025 Hz) using a speech synthesis software (Oshaberi-mate, Fujitsu, Tokyo) that converts J apanese written texts into sound waveforms. With this software, target and nontarget stimuli sounded natural for the prosody of speech in J apanese. The speech stimuli for both targets and nontargets were presented at the maximum intensity of $67 \mathrm{~dB}$ SPL (sound pressure level), measured by a sound level meter (NL-14, Rion, Tokyo) at the tip of the tube used in the experiment. The duration of each stimulus ranged from 400 to $700 \mathrm{~ms}$. The target for nonspeech sounds was a $400-\mathrm{Hz}$ sine wave with low-passed white noise at $400 \mathrm{~Hz}$ (covering up to the range of the fundamental frequencies), and the nontarget was the white noise alone. Each nonspeech stimulus was delivered at $62 \mathrm{~dB}$ SPL for $600 \mathrm{~ms}$. Auditory stimuli were presented to the subject's ears every $1 \mathrm{~s}$ through separate tubes (inner diameter: $9 \mathrm{~mm}$, length: $5.9 \mathrm{~m}$ ), and the scanning sounds were confined within the interstimulus-interval of 300-600 ms.

\section{Tasks}

The behavioral tasks used here were a control condition (CON), in which nonspeech sounds were presented diotically, and two listening conditions for speech sounds (Fig. 1): DIO and DIC. The subject was asked to respond to a target stimulus by pressing a bulb attached to a pneumatic switch as fast as possible with a right hand, while ensuring correct responses. The rate of bulb pressing was 0.5 in all conditions. Under the DIO condition, identical stimuli (either targets or nontargets) were presented to both ears simultaneously. Target phrases were presented in the order of an original story with interventions of nontargets. Nontargets were presented before their corresponding targets appeared. The number of nontargets intervening between two targets varied from zero to two randomly (mean $=1$, SD $=0.92$ ). Therefore, the subjects cannot predict exactly when the target phrase appears in a sequence. Under the DIC condition, either a pair of target and nontarget or that of two different nontargets was simultaneously presented to different ears. The side of ears, to which a target was presented, was randomized, and the rate of target phrase occurrence (1/4 for each ear on average) was equated between two 


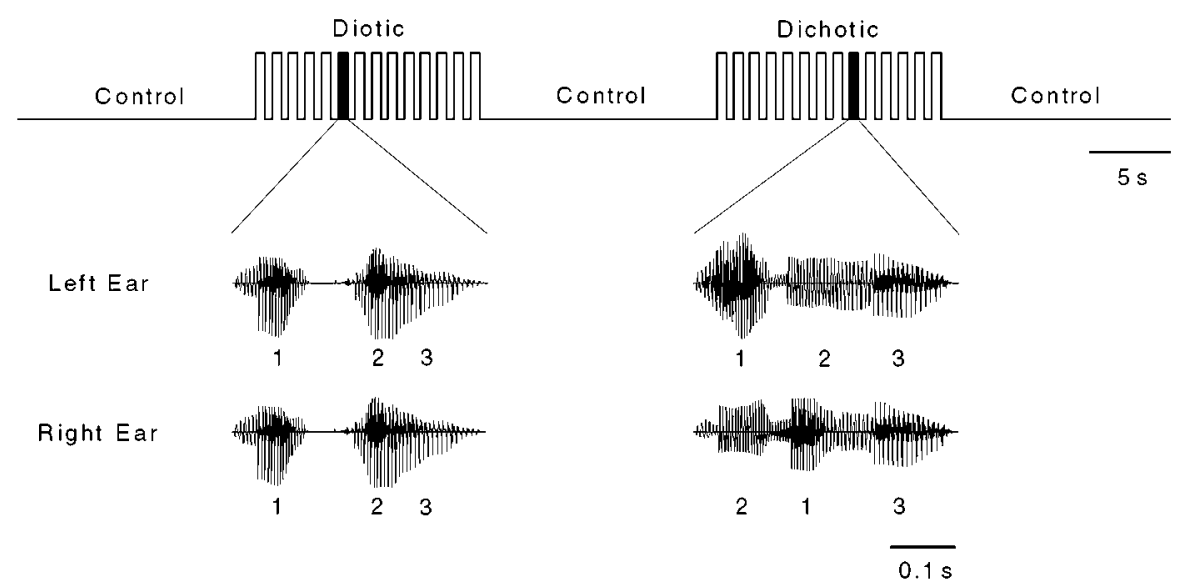

FIG. 1. Diotic and dichotic listening stimuli for speech recognition. A story was read in J apanese and divided into phrases with three or more syllables. Numbers below sound waveforms denote the original order of syllables in each story phrase. As a nontarget stimulus, we changed the sequence of syllables of the phrase coming next in the story. An example of a nontarget is shown in the right panel (2-1-3), this nontarget was formed from an original story phrase (1-2-3). These jumbled stimuli conform to the rules of $\mathrm{J}$ apanese phonotactics but have no meaning.

ears. The subject has to choose one side with a target, and to attend that target selectively. Therefore, in addition to the recognition of speech sounds required by the DIO condition, the DIC condition also demands selective attention to target stimuli in either ear. Because the rate of target phrase occurrence was equated between DIO and DIC conditions, information rate was balanced for these conditions in terms of relevant targets. In a single session (238 s), DIO and DIC blocks were presented alternately in the order of CON-DIOCON-DIC-CON... (Fig. 1), and each session consisted of nine CON blocks, four DIO blocks, and four DIC blocks (14 s each). Both accuracy and RT were measured on-line, and the stimulus presentation and behavioral data collection were controlled by a PC-LabVIEW system (National Instruments, Austin, TX).

\section{fMRI Data Acquisition and Analysis}

The present study was performed using a 1.5-Tesla fMRI system (MRH-1500, Hitachi Medical Corp., Tokyo). We scanned over four horizontal slices of 8-mm thickness, covering between 0 and $32 \mathrm{~mm}$ from the AC-PC line, with a gradient echo echoplanar imaging (EPI) sequence (TR $=2 \mathrm{~s}, T E=22 \mathrm{~ms}$, half scanning, flip angle $=90^{\circ}$, field of view $=384 \times 384 \mathrm{~mm}^{2}$, resolution $=3 \times 3 \mathrm{~mm}^{2}$ ) (Stehling et al., 1991; Sakai et al., 1995a, 1995b). The distance from AC-PC lineto the top of the cerebral cortex was $71.2 \pm 4.2 \mathrm{~mm}$ (mean \pm SD) in tested subjects. In a single scanning session, we obtained 119 images for each slice; we tested at least eight sessions per subject. During the same session as fMRI scanning, we obtained structural images by using a spin echo sequence $(T R=500 \mathrm{~ms}$, TE $=20 \mathrm{~ms}$, flip angle $=90^{\circ}$, field of view $=384 \times 384 \mathrm{~mm}^{2}$, resolution $=1.5 \times 1.5 \times 8 \mathrm{~mm}^{3}$ ) at the sameslice positions as those of EPI images. Shim currents were carefully adjusted to minimize distortions of EPI images from structural images.

Analyses of fMRI time-series data were first done on a single-subject basis, using in-house software (Sakai et al., 1995a). Time-series data of each voxel were converted to percent signal changes from the initial CON block and corrected for baseline using linear fitting to data throughout all CON periods with a hemodynamic delay of $6 \mathrm{~s}$ (a delay of $4 \mathrm{~s}$ was used for one subject to obtain higher signal-to-noise ratio). They were then averaged for multiple sessions after correction for head movements between scans, without any spatial or temporal smoothing. We estimated the activation under the DIO and DIC conditions using the following two types of t- tests for each voxel: (DIO + DIC) vs CON ( $\mathrm{t}>2.6, \mathrm{P}<0.005, \mathrm{df}=135$ ( 17 blocks $\times 8$ sessions $1)$, one-tailed, uncorrected for multiple comparisons) combining DIO and DIC before comparison with CON, and DIC vs DIO (t $>2.3, \mathrm{P}<0.01$ ) as direct comparison between two experimental conditions. The significance level for each activated region after Bonferroni correction was $\mathrm{P}<0.02$ and $\mathrm{P}<0.04$, respectively. We use abbreviations of these t maps as follows: $t$ map $(\alpha)$ for $(\mathrm{DIO}+\mathrm{DIC})$ vs CON, and t map ( $\beta$ ) for DIC vs DIO. We set a more stringent statistical threshold for the t map $(\alpha)$ with larger contrast. We identified an activated region as a cluster with at least four contiguous voxels of $t>2.6$ and $t>2.3$ that contain a local maximum of t values in t map $(\alpha)$ and t map $(\beta)$, respectively.

In case four voxels in t map $(\alpha)$ overlapped with four voxels in t map $(\beta)$ for more than one voxel, the four voxels in the latter t map were used for analysis. To compare cortical responses across conditions, signal changes from the baseline level were averaged among four voxels with the highest $t$ values in a region; we did not compare the spatial extents of activated regions. 
TABLE 1

Multiple Auditory Areas Showing Responses to Speech Sounds

\begin{tabular}{|c|c|c|c|c|c|c|c|c|c|}
\hline Region & t map & BA & Hemisphere & $\mathrm{n}$ & $x$ & $y$ & z & DIC vs DIO & DSI \\
\hline \multirow[t]{2}{*}{ A1 } & $\alpha / \beta$ & 41 & $\mathrm{~L}$ & 6 & $-44 \pm 5$ & $-13 \pm 6$ & $7 \pm 4$ & $9.1^{*}$ & $0.35 \pm 0.22$ \\
\hline & & & $\mathrm{R}$ & 3 & $43 \pm 4$ & $-13 \pm 3$ & $10 \pm 1$ & 5.2 & $0.17 \pm 0.13$ \\
\hline \multirow[t]{2}{*}{$\mathrm{A} 2 \mathrm{~m}$} & $\beta$ & 42 & $L$ & 7 & $-44 \pm 2$ & $-27 \pm 5$ & $6 \pm 3$ & $18.3^{* *}$ & $0.52 \pm 0.09 \dagger$ \\
\hline & & & $\mathrm{R}$ & 6 & $43 \pm 3$ & $-21 \pm 4$ & $9 \pm 3$ & $9.8^{*}$ & $0.60 \pm 0.19 \dagger$ \\
\hline \multirow[t]{2}{*}{ A2I } & $\alpha / \beta$ & 42 & $\mathrm{~L}$ & 7 & $-53 \pm 2$ & $-15 \pm 6$ & $6 \pm 2$ & 3.1 & $0.32 \pm 0.09$ \\
\hline & & & $\mathrm{R}$ & 6 & $52 \pm 4$ & $-9 \pm 5$ & $7 \pm 3$ & 1.7 & $0.24 \pm 0.19$ \\
\hline \multirow[t]{2}{*}{ STa } & $\alpha / \beta$ & 42 & $\mathrm{~L}$ & 6 & $-61 \pm 3$ & $-16 \pm 6$ & $5 \pm 2$ & 0.9 & $0.19 \pm 0.11$ \\
\hline & & & $\mathrm{R}$ & 3 & $59 \pm 3$ & $-11 \pm 3$ & $8 \pm 3$ & 0.8 & $0.30 \pm 0.01$ \\
\hline \multirow[t]{2}{*}{$\mathrm{PT} \alpha$} & $\alpha$ & $42 / 22$ & $\mathrm{~L}$ & 7 & $-52 \pm 7$ & $-29 \pm 8$ & $13 \pm 4$ & 2.4 & $0.25 \pm 0.11$ \\
\hline & & & $\mathrm{R}$ & 3 & $53 \pm 3$ & $-25 \pm 3$ & $16 \pm 4$ & 2.8 & $0.30 \pm 0.16$ \\
\hline \multirow[t]{2}{*}{$\mathrm{PT} \beta$} & $\beta$ & $42 / 22$ & $\mathrm{~L}$ & 6 & $-52 \pm 4$ & $-30 \pm 5$ & $13 \pm 3$ & $15.5^{* *}$ & $0.72 \pm 0.13 \dagger$ \\
\hline & & & $\mathrm{R}$ & 5 & $53 \pm 3$ & $-18 \pm 7$ & $14 \pm 2$ & $15.5^{* *}$ & $0.71 \pm 0.12 \dagger$ \\
\hline \multirow[t]{2}{*}{$\mathrm{ST} \alpha$} & $\alpha$ & 22 & $\mathrm{~L}$ & 6 & $-56 \pm 5$ & $-43 \pm 5$ & $7 \pm 4$ & 0.1 & $0.11 \pm 0.31$ \\
\hline & & & $\mathrm{R}$ & 7 & $50 \pm 4$ & $-30 \pm 11$ & $7 \pm 3$ & 1.4 & $0.20 \pm 0.10$ \\
\hline \multirow[t]{2}{*}{$\mathrm{ST} \beta$} & $\beta$ & 22 & $\mathrm{~L}$ & 7 & $-59 \pm 3$ & $-39 \pm 11$ & $8 \pm 4$ & $9.8^{*}$ & $0.61 \pm 0.08 \dagger$ \\
\hline & & & $\mathrm{R}$ & 7 & $51 \pm 4$ & $-34 \pm 5$ & $8 \pm 4$ & $9.8^{*}$ & $0.75 \pm 0.12 \dagger$ \\
\hline \multirow[t]{2}{*}{ SMG } & $\alpha$ & 40 & $\mathrm{~L}$ & 5 & $-58 \pm 4$ & $-41 \pm 4$ & $20 \pm 4$ & 3.0 & $0.32 \pm 0.12$ \\
\hline & & & $\mathrm{R}$ & 2 & $53 \pm 6$ & $-38 \pm 8$ & $26 \pm 2$ & 0.1 & $0.01 \pm 0.25$ \\
\hline
\end{tabular}

Note. For the definition of each region, see text. Column t map refers to either the t map ( $\alpha)$ of (DIO + DIC) vs CON or the t map ( $\beta)$ of DIC vs DIO, which was used for identification of each region. In the case of $\alpha / \beta$, a region was identified either in the t map ( $\alpha$ ) or in the t map $(\beta)$ (see Fig. 3). $n$ is the number of subjects who showed significant activation. Columns $x, y$, and $z$ correspond to the coordinates (mean \pm SD) of centers of activated regions, stated as millimeters from the anterior end of the AC-PC line, after affine transformation of individual hemispheres to the standard brain (Talairach and Tournoux, 1988). Column DIC vs DIO refers to F values obtained by $F$ test between the $\mathrm{DIO}$ and DIC conditions in each region: $\mathrm{F}(1,2 \mathrm{n}-2)$. Single $\left(^{*}\right)$ and double $\left(^{* *}\right)$ asterisks denote statistical significance at $\mathrm{P}<0.05$ and $\mathrm{P}<$ 0.005 , respectively. Column DSI refers to DSI values (mean \pm SD) in each region: DSI $=(D I C-D I O) / D I C$. †A mean DSI value above 0.5 . Abbreviations: A1, primary auditory cortex; A2, secondary auditory cortex; A2I, lateral secondary auditory cortex; A2m, medial secondary auditory cortex; BA, Brodmann's area; DIC, dichotic listening; DIO, diotic listening; DSI, DIC selectivity index; PT, planum temporale; SMG, supramarginal gyrus; ST, superior temporal gyrus and sulcus; STa, anterior superior temporal gyrus.

When two or more clusters were identified in onet map as separate but still within the same predefined anatomical region, signal changes were averaged among these clusters. These clusters might be candidates for further functional parcellation, but it is possible that slicing undulate gyri resulted in apparently separated clusters.

For signal changes in each region identified by t map $(\alpha)$ and/or $t$ map $(\beta)$, we performed $F$ test between the DIO and DIC conditions, thereby pooling the data from all subjects (Tables 1 and 2). Furthermore, in order to estimate the degree of DIC selectivity in each region, we calculated DIC selectivity index (DSI) using the signal changes from baseline under DIO and DIC conditions: DSI = (DIC - DIO)/DIC.

\section{Anatomical Identification}

A three-dimensional structural image of a whole brain of each subject was obtained using a gradient

\section{TABLE 2}

Frontal Regions Showing Responses to Speech Sounds

\begin{tabular}{|c|c|c|c|c|c|c|c|c|c|}
\hline Region & t map & BA & Hemisphere & $\mathrm{n}$ & $x$ & $y$ & $z$ & DIC vs DIO & DSI \\
\hline \multirow[t]{2}{*}{$\mathrm{IF} \alpha$} & $\alpha$ & $44 / 45$ & $\mathrm{~L}$ & 5 & $-46 \pm 7$ & $17 \pm 7$ & $14 \pm 9$ & $13.2^{*}$ & $0.26 \pm 0.08$ \\
\hline & & & $\mathrm{R}$ & 5 & $49 \pm 7$ & $15 \pm 4$ & $19 \pm 10$ & 2.7 & $0.35 \pm 0.13$ \\
\hline \multirow[t]{2}{*}{$I F \beta$} & $\beta$ & $44 / 45$ & $\mathrm{~L}$ & 6 & $-43 \pm 4$ & $15 \pm 6$ & $22 \pm 4$ & $6.7 *$ & $0.69 \pm 0.17 \dagger$ \\
\hline & & & $\mathrm{R}$ & 7 & $39 \pm 6$ & $17 \pm 3$ & $20 \pm 4$ & $30.4^{* *}$ & $0.78 \pm 0.20 \dagger$ \\
\hline \multirow[t]{2}{*}{ Anterior insula } & $\alpha$ & - & $\mathrm{R}$ & 3 & $28 \pm 1$ & $14 \pm 2$ & $12 \pm 7$ & 6.2 & $0.54 \pm 0.08 \dagger$ \\
\hline & $\beta$ & - & $\mathrm{R}$ & 4 & $36 \pm 2$ & $16 \pm 6$ & $14 \pm 9$ & $25.2^{* *}$ & $0.74 \pm 0.16 \dagger$ \\
\hline \multirow[t]{2}{*}{ Precentral gyrus } & $\alpha$ & $4 / 6$ & L & 3 & $-55 \pm 2$ & $-2 \pm 7$ & $14 \pm 4$ & 0.001 & $-0.02 \pm 0.15$ \\
\hline & & & $\mathrm{R}$ & 3 & $53 \pm 4$ & $2 \pm 2$ & $16 \pm 2$ & 0.7 & $0.20 \pm 0.15$ \\
\hline
\end{tabular}

Note. For the definition of each region, see text. The regions that were identified in more than two subjects are listed here. Single $(*)$ and double (**) asterisks denote statistical significance at $\mathrm{P}<0.05$ and $\mathrm{P}<0.005$, respectively. †A mean DSI value above 0.5 (see Table 1 ). Abbreviations: IF, inferior frontal gyrus and sulcus. 


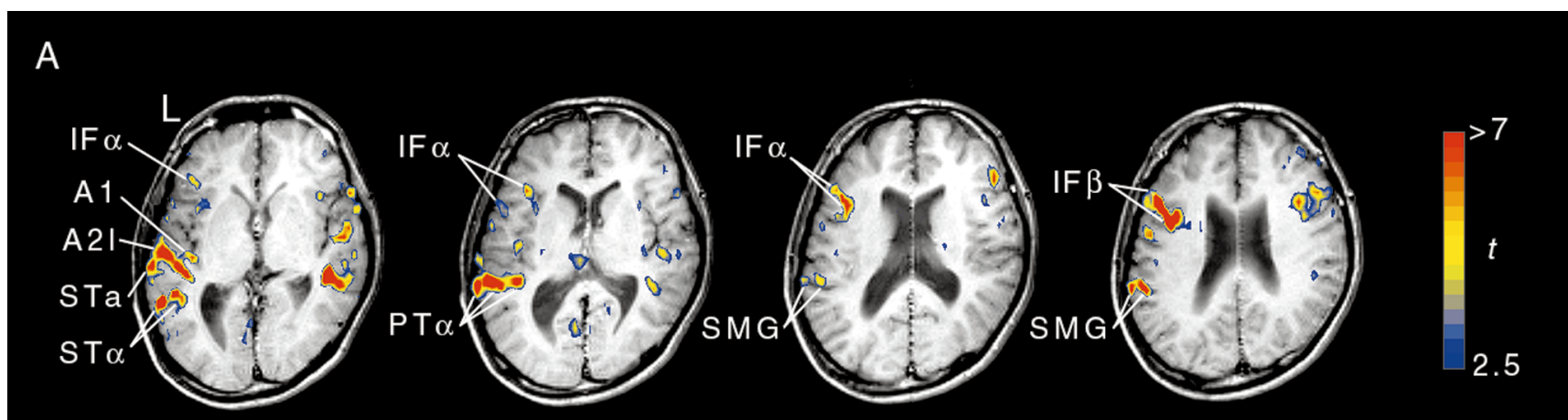

B
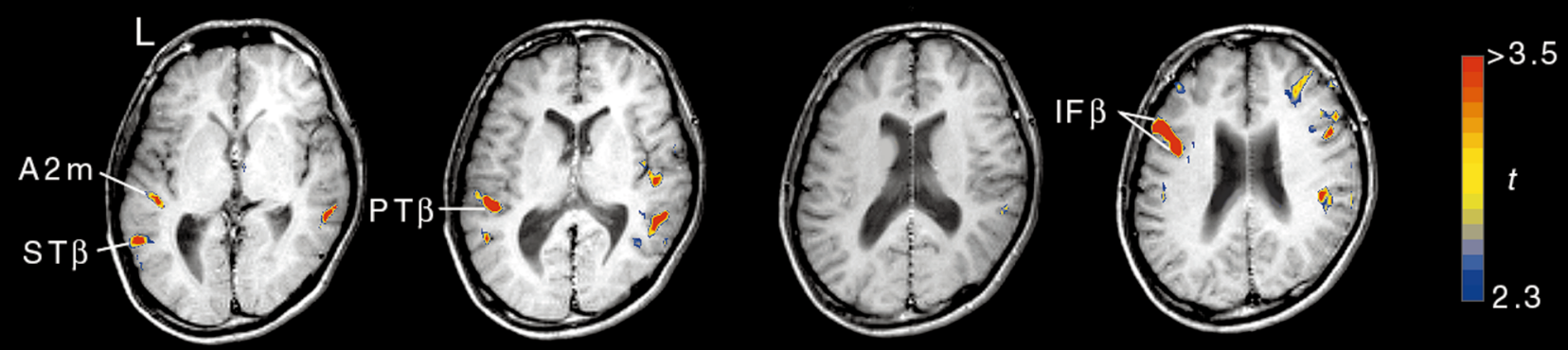

2

A

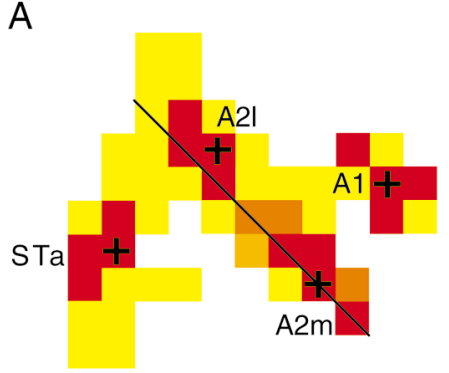

D

\section{3}

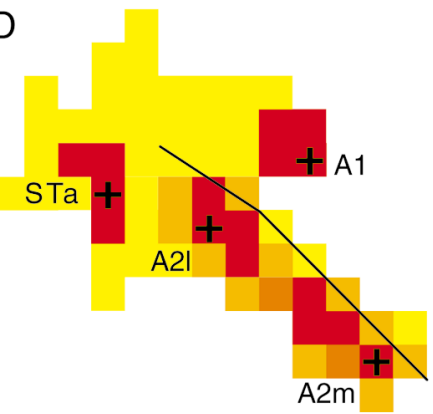

B

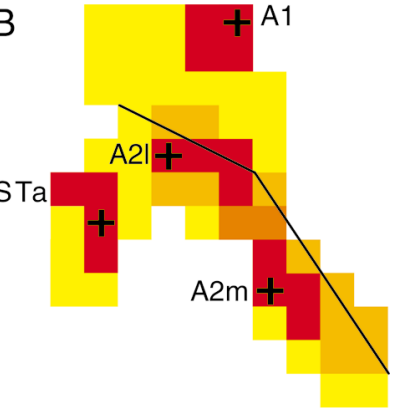

E

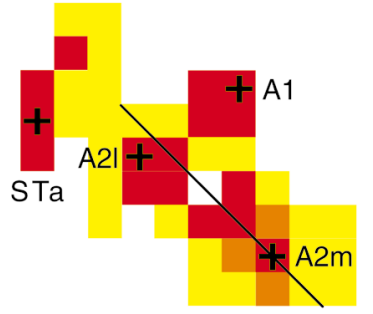

C

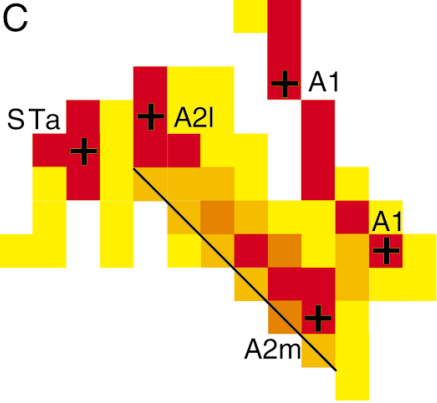

F

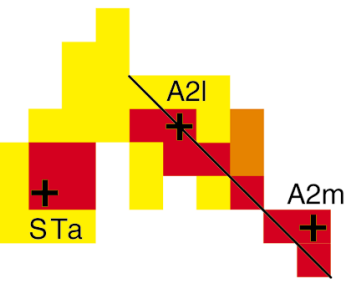

G

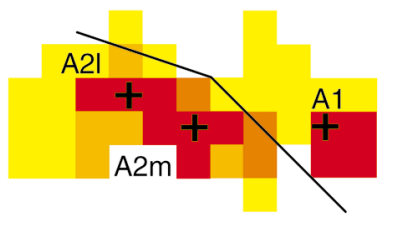

FIG. 2. Representative horizontal slices showing multiple auditory areas in one subject. (A) A series of $t$ maps ( $\alpha$ ) of (DIO + DIC) vs CON. (B) A series of t maps $(\beta)$ of DIC vs DIO. Color bars denote t values for each comparison. The left side of the brain is shown left in each horizontal slice. The centers of the slices are $z=4,12,20,28$ (see Table 1) from left panel to right. Anatomical and functional images in this figure were interpolated bilinearly. Multiple activated regions were found in the auditory and language-related areas, mostly in the left hemisphere of this subject.

FIG. 3. Spatial relationship among $A 1, A 2 m, A 2 l$, and $S T a$ in the left hemisphere. Each panel (A-G) represents individual subjects. The left side of the brain is shown left in the individual horizontal slices. Voxel size, $3 \times 3 \mathrm{~mm}^{2}$. The area shown in (A) is a portion of the t maps shown in Fig. 2 (the leftmost column). Yellow voxels are significant voxels in the t map ( $\alpha)$ of (DIO + DIC) vs CON. Significant voxels in the t map ( $\beta$ ) of DIC vs DIO are superimposed and shown in yellow-orange. All of the yellow-orange voxels fall onto yellow voxels. The four contiguous voxels with the highest $t$ values, which were chosen for each region in either the t map $(\alpha)$ or the t map ( $\beta$ ), are shown in red. To show the spatial extent of activation in A2m, two or three adjacent voxels with higher t values (DIC vs DIO) are shown in orange. Plus (+) indicates a local maximum of $t$ values in each activated region. Solid lines represent Heschl's sulcus in individual subjects, and it is clear that A2m and A2l extend al ong HS. Note that red voxels in $\mathrm{A} 1, \mathrm{~A} 2 \mathrm{~m}, \mathrm{~A} 2 \mathrm{l}$, and $\mathrm{ST}$ a are well separated and that their spatial relationship is consistent among all subjects. 
echo sequence $(\mathrm{TR}=30 \mathrm{~ms}, \mathrm{TE}=8 \mathrm{~ms}$, flip angle $=$ $60^{\circ}$, field of view $=384 \times 384 \mathrm{~mm}^{2}$, resolution $1.5 \times$ $1.5 \times 3 \mathrm{~mm}^{3}$ ) in a separate session. For each subject, the horizontal slices of structural and functional images were cor egistered by translation and rotation onto the subject's three-dimensional structural image. After selecting activated clusters on t maps (see fMRI Data Acquisition and Analysis), we labeled those activated clusters as belonging to a particular region, based on the three-dimensional structures of sulci and gyri with the following definitions of the boundaries: A1 (primary auditory area), the anteromedial part of Heschl's gyrus (HG); A2 (secondary auditory area), the regions located along Heschl's sulcus (HS) and within BA 42; PT (planum temporale), the area that spans from the posterolateral border of A2 to the posterior end of the supratemporal plane (Steinmetz et al., 1989; M offat et al., 1998); ST (superior temporal gyrus and sulcus), the lateral area that spans from anterior end of the superior temporal sulcus (STS) to ascending ramus of STS, as well as STS itself; SMG (supramarginal gyrus), the area that lies between the postcentral sulcus and the intermediate sulcus of J ensen; IF (inferior frontal gyrus and sulcus), pars triangularis and pars opercularis; anterior insula, the area that lies anterior to the central insular sulcus; precental gyrus, the area that lies between the precentral sulcus and the central sulcus. After affine transformation of individual hemispheres to the standard brain (Talairach and Tournoux, 1988), the average coordinates of each region among all subjects was calculated (Tables 1 and 2).

\section{RESULTS}

\section{Task Performance}

The accuracy under the DIO and DIC conditions was $88 \pm 4.5$ and $77 \pm 5.1 \%$ (mean \pm SD, $n=7$ ), respectively. The errors under these conditions contained time-out errors, as the current paradigm required the subject to respond within $980 \mathrm{~ms}$ from the onset of the stimulus. RT under the DIO and DIC conditions was $910 \pm 41$ and $920 \pm 26 \mathrm{~ms}$, respectively. Although accuracy was different between these conditions $(F(1,12)=16.6, P<0.005)$, there was no significant difference in RT $(F(1,12)=0.39, P>0.5)$.

\section{Functional Parcellation of Auditory Cortex}

We identified multiple regions that were activated under the DIO and DIC conditions (Fig. 2). Here we will focus on auditory areas first, describing activated regions in the frontal lobe later. In the $t \operatorname{map}(\alpha)$ of $(\mathrm{DIO}+\mathrm{DIC})$ vs CON, activation was observed in A1, A2 (both lateral and medial portions), PT, ST, and SMG (Fig. 2A). In the t map ( $\beta$ ) of DIC vs DIO, on the other hand, we identified regions in A2 (medial portion), PT, and ST that responded more prominently to the DIC condition (Fig. 2B). In A2, the medial portion clearly showed DIC selectivity, though this selectivity was not apparent in the lateral portion. These lateral and medial regions correspond to two local maxima of t values along HS (Fig. 3), which we call A2l (lateral A2) and A2m (medial A2), respectively. We identified STa (anterior ST) as an activated region just posterior to HS and on the lateral surface of the anterior superior temporal gyrus. Functionally distinct subareas were also found in PT and ST; some regions were identified in the t map ( $\beta$ ): PT $\beta$ and ST $\beta$, while other areas were identified only in the t map $(\alpha)$ : PT $\alpha$ and $\mathrm{ST} \alpha$. The location of each region and the corresponding BA are shown in Table 1. In the left hemisphere, we observed these regions in at least five subjects. Although some regions in the right hemisphere were activated in a smaller number of subjects, all the re gions identified in the left hemisphere were also found in the right hemisphere at similar coordinates.

\section{Spatial Relationship among Early Auditory Areas}

We observed clear spatial segregation between $A 1$ and A2 (Figs. 2A and 3). A2 was located either just anterior or posterior to HS, depending on the subjects (Fig. 3). This individual variation agrees with previous anatomical reports, which have shown that the boundary between $A 1$ and $A 2$ is not always HS itself, but is sometimes anterior to HS (Rademacher et al., 1993; Hutsler and Gazzaniga, 1996). Though A2m and A2I were activated both under DIO and DIC conditions, we found functional differentiation between $A 2 m$ and $A 2$ I as described above. Further, we confirmed that the spatial relationship between A2m and A2I was consistent among all subjects (Fig. 3); A2m located posteromedial to A2I (Table 1), and the difference in $x$ and $y$ coordinates between $\mathrm{A} 2 \mathrm{~m}$ and $\mathrm{A} 2 \mathrm{l}$ was significant in both left hemisphere $(x: F(1,12)=54.4, P<0.0001 ; y$ : $F(1,12)=13.4, P<0.005)$ and right hemisphere $(x:$ $F(1,10)=16.8, P<0.005 ; y: F(1,10)=15.6, P<0.005)$. STa was consistently located just lateral to $A 2 \mathrm{l}$, and the difference between $\mathrm{A} 2 \mathrm{I}$ and STa in $\mathrm{x}$ coordinates was significant in both hemispheres (left: $F(1,11)=$ 38.9, $\mathrm{P}<0.0001$; right: $\mathrm{F}(1,7)=6.6, \mathrm{P}<0.05)$.

\section{Differential Activation under the DIO and DIC Conditions}

Representative temporal signal changes in the auditory areas are shown in Fig. 4. Signal changes in all auditory regions observed and difference in conditions are summarized in Fig. 5 and Table 1. The DIC condition elicited larger mean signal changes than the DIO condition in all regions. The difference between these two conditions was most prominent in $\mathrm{A} 2 \mathrm{~m}, \mathrm{PT} \beta$, and ST $\beta$ for both hemispheres (Figs. $5 B$ and $5 E$, see also Figs. $4 \mathrm{~A}$ and $4 \mathrm{C}$ for left $\mathrm{A} 2 \mathrm{~m}$ and ST $\beta$ ), whereas A2I, $\mathrm{STa}, \mathrm{PT} \alpha, \mathrm{ST} \alpha$, and SMG showed smaller response difference in both hemispheres (Figs. $5 \mathrm{C}$ and $5 \mathrm{~F}$, see 
A

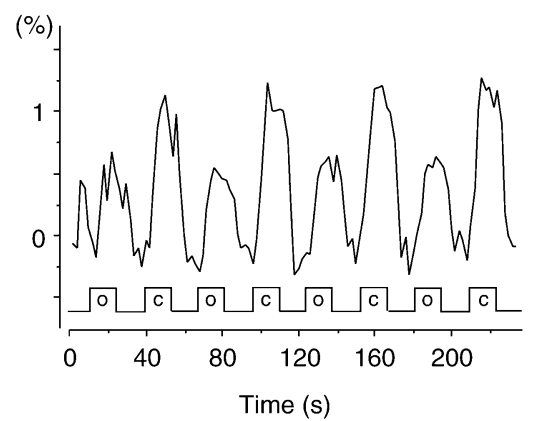

C

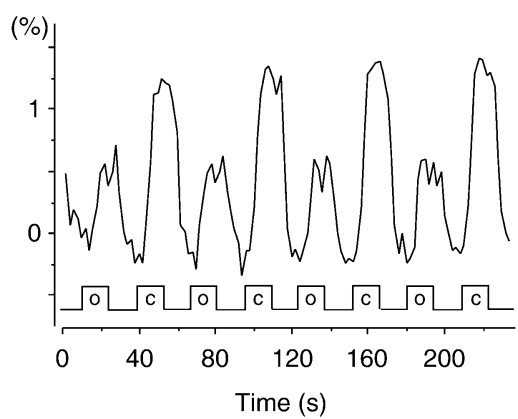

B

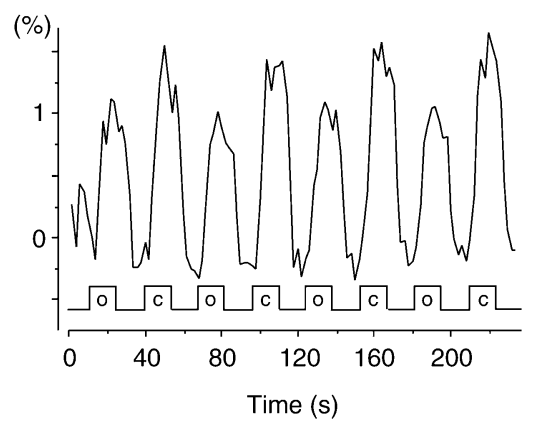

D

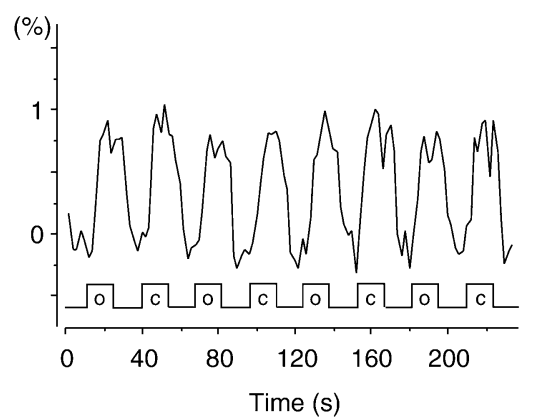

FIG. 4. Mean time-series for representative regions in the study. (A) Temporal percent signal change in left $A 2 m$, averaged among all subjects. (B) Temporal signal change in left A2I. (C) Temporal signal change in left ST $\beta$. (D) Temporal signal change in left ST $\alpha$. Note the difference between signal changes under the DIO condition (o) and the DIC condition (c) in each region. All panels are shown with the same ordinate scale.

also Figs. 4B and 4D for left $A 21$ and $S T \alpha$ ). We confirmed that the difference between the DIO and DIC conditions was not due to habituation or repetition effects, based on the observation of no signal difference between the second blocks and the fourth blocks under both conditions for A2m (DIO:t (6) $=-1.0, \mathrm{P}>0.1$; DIC: $\mathrm{t}(6)=-2.1, \mathrm{P}>0.05$; paired t test, two-tailed), PT $\beta$ (DIO: t (5) = -1.4, P > 0.1; DIC: t (5) $=-1.3, \mathrm{P}>$ 0.1 ), and ST $\beta$ (DIO: t (6) $=-0.33, \mathrm{P}>0.5$; DIC: $\mathrm{t}(6)=$ $-1.3, \mathrm{P}>0.1$ ) in the left hemisphere.

The differential signal changes in multiple auditory areas were further confirmed by an analysis of variance (ANOVA) with three variables (condition $\times$ region $\times$ hemisphere). We found a significant interaction between regions ( 9 levels of the region factor: $A 1, A 2 m$, A2I, STa, PT $\alpha, \mathrm{PT} \beta, \mathrm{ST} \alpha, \mathrm{ST} \beta, \mathrm{SMG})$ and conditions $(F(8,170)=2.0, P<0.05)$, indicating that responses in some regions were more prominently enhanced by the DIC condition than in the other regions. This result demonstrates clear functional differentiation among multiple auditory areas. Although the location of activated regions is matched for both hemispheres (Table $1)$, the signal changes in the left regions were significantly larger than those in the right regions (a main effect of hemispheres, $F(1,170)=5.1, P<0.05)$. The activated regions shown in Fig. 2 also demonstrate the left dominance in a representative subject. There was neither a main effect of subjects $(F(6,176)=2.1, P>$
$0.05)$ nor interactions between subjects and other factors (conditions: $\mathrm{F}(6,176)=0.42, \mathrm{P}>0.5$; hemispheres: $F(6,176)=1.3, P>0.1)$.

\section{Activated Regions in the Frontal Lobe}

Besides the auditory areas, we also found activated regions in the frontal lobe; left and right IF, the right anterior insula, and the left and right precentral gyrus. Similar to PT and ST, we identified activated regions in IF in the $t$ map $(\alpha)$ and in the $t$ map $(\beta)$, which we named as IF $\alpha$ and IF $\beta$, respectively. Signal changes in these regions are shown in $\mathrm{Figs}$. 5B, 5C, 5E, and 5F. The location of each frontal region and corresponding $B A$, as well as the difference in conditions, are shown in Table 2. We observed significant difference in conditions for left IF $\beta$, right IF $\beta$, left IF $\alpha$, and the right anterior insula $(\beta)$ (Table 2 ). These results suggest that IF and the right anterior insula are further subdivided into functionally distinct regions, and a functional role of the anterior insula may be different between hemispheres in our paradigms.

\section{Spatial Relationship among Language Areas}

Though the spatial relationship among A2m, A2l, and STa was consistent among subjects, the spatial relationship among activated regions in $\mathrm{PT}, \mathrm{ST}$, and I F was not apparent. According to the definition of indi- 
A

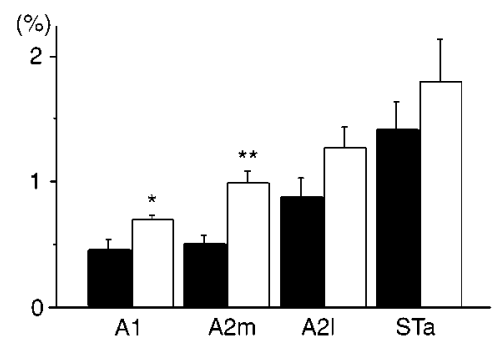

D

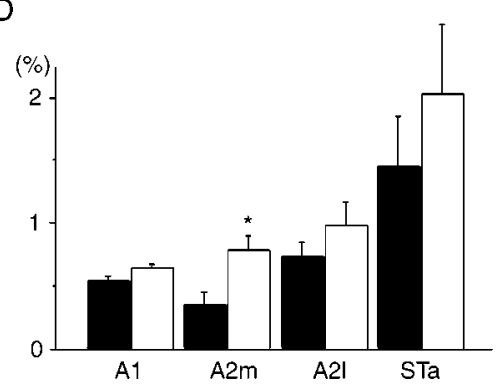

B

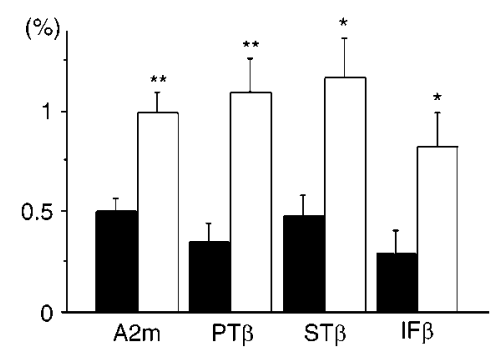

$E$

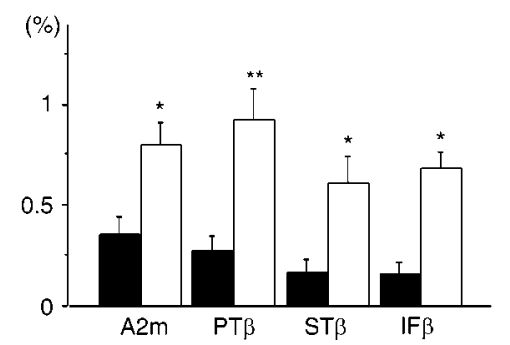

C

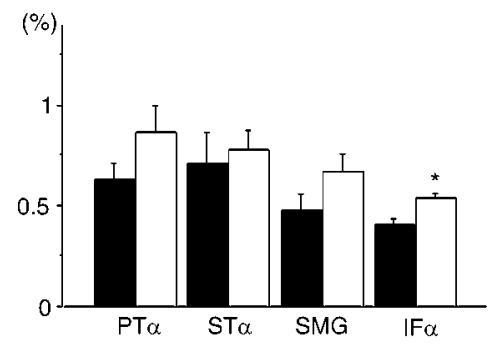

$\mathrm{F}$

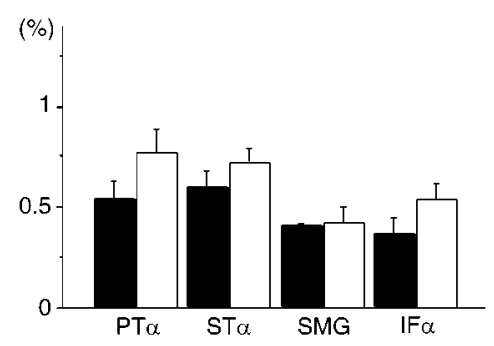

FIG. 5. Differential responses under the DIO and DIC conditions in multiple auditory areas. Histogram comparing the percent signal changes (mean \pm SEM of subjects) for each auditory condition vs CON is shown in each region. Filled and open bars denote signal changes under DIO and DIC, respectively. (A-C) Regions in the left hemisphere. (D-F) Regions in the right hemisphere. Regions that show different response patterns are shown in separate panels. (A and D) Signal changes in A1, A2m, A21, and STa; areas with close anatomical relationships. They show progressive signal increases under the DIO and DIC conditions. A and D are shown with the same ordinate scale. (B and E) Signal changes in A2m, PT $\beta$, ST $\beta$, and IF $\beta$; areas with DIC-selective responses. A2m is shown again here for comparison. (C and F) Signal changes in PT $\alpha, \mathrm{ST} \alpha, \mathrm{SMG}$, and IF $\alpha$; areas with comparable responses to the DIO and DIC conditions. Panels B, C, E, and F are shown with the same ordinate scale. Single $\left(^{*}\right)$ and double $\left(^{* *}\right)$ asterisks denote statistical significance at $\mathrm{P}<0.05$ and $\mathrm{P}<0.005$, respectively (see Tables 1 and 2).

vidual regions (see Materials and Methods), PT $\alpha, \mathrm{PT} \beta$, ST $\alpha$, ST $\beta$, IF $\alpha$, and IF $\beta$ were clearly segregated. Each of these regions was not always unitary but consisted of multiple clusters within a single subject, as shown in Fig. 2, while $A 2 m, A 21$, and STa were unitary regions. The number and locations of these clusters, as well as the spatial relationship between PT $\alpha$ and $\mathrm{PT} \beta, \mathrm{ST} \alpha$ and ST $\beta$, or IF $\alpha$ and IF $\beta$, were not consistent among subjects, which may be due to individual variability of functional organization or structural complexity in these areas. It has been reported that PT is structurally highly variable even within right-handed individuals (Steinmetz, 1996; Westbury et al., 1999), and that IF also showed individual variation in spatial relationship of functional subregions (Kim et al., 1997). Mean coordinates of centers of PT $\alpha$ and PT $\beta, \mathrm{ST} \alpha$ and ST $\beta$, or IF $\alpha$ and IF $\beta$ were almost identical (Tables 1 and 2 ), in spite of the fact that each of multiple clusters was separated. The present study is significant in its identification of regions in PT, ST, and IF with two distinct response patterns in individual subjects.

\section{Classification of Auditory and Language Areas}

In order to estimate the degree of DIC selectivity in each region, we used DSI (see Materials and Methods). Among regions with activation found in both hemi- spheres, A2m, PT $\beta, \mathrm{ST} \beta$, and IF $\beta$ were the only regions with DSI above 0.5 (Tables 1 and 2), corresponding to our identification of these DIC-selective regions based on the t map ( $\beta$ ). According to an ANOVA (region $\times$ hemisphere) for $D S I$ of these regions, there was a main effect of regions $(F(3,43)=3.8, P<0.05)$ and hemispheres $(F(1,43)=4.1, P<0.05)$ without a significant interaction $(F(3,43)=0.59, P>0.5)$, and $A 2 m$ showed a significantly smaller DSI (Fisher's PLSD, P $<0.05$ ). This result suggests progressive processing of DICselective information among these regions. In contrast, there was no main effect of regions $(F(3,32)=2.1, P>$ $0.1)$ and hemispheres $(F(1,32)=0.11, P>0.5)$ among $\mathrm{PT} \alpha, \mathrm{ST} \alpha, \mathrm{SMG}$, and I $\mathrm{F} \alpha$ regions without a significant interaction $(F(3,32)=2.2, P>0.1)$ in both hemispheres.

Among the multiple auditory areas identified in the t maps, A1, A2m, A2l, and STa have close anatomical relationships (Figs. 2 and 3). Signal changes for the DIO and DIC conditions showed a progressive increase in the mediolateral order, from A1 to STa in both hemispheres (Figs. 5A and 5D). While $A 1$ and $A 2 m$ showed greater responses to the DIC condition than to the DIO condition, the difference between the two conditions was attenuated in A2l and STa, the more lateral regions. According to an ANOVA (region $\times$ hemi- 
sphere) for DSI of these regions, there was a strong main effect of regions $(F(3,36)=12.1, P<0.0001)$ with neither a main effect of hemispheres $(F(1,36)=0.15$, $P>0.5)$ nor a significant interaction $(F(3,36)=2.1$, $P>0.1)$. Al showed a significantly smaller DSI than A2m (Fisher's PLSD, P $<0.0005$ ). Furthermore, there was a significant decrease in DSI along the same spatial progression of left A2m, A2l, and STa $(P<0.05)$.

\section{DISCUSSION}

\section{Functional Differentiation in Multiple Auditory Areas}

The present study clearly demonstrates that the DIO and DIC conditions modulate cortical responses differentially among multiple auditory areas. Some areas showed greater responses to the DIC condition than to the DIO condition, while other areas did not show such a difference. These contrasting response patterns suggest functional differentiation among multiple auditory areas. Furthermore, we found functional differentiation within A2. The DIC selective response was observed in the medial portion of $A 2(A 2 m)$ as well as in restricted regions of $\mathrm{PT}$ and $\mathrm{ST}$ (PT $\beta$ and $\mathrm{ST} \beta$, respectively), whereas the lateral portion of $A 2$ (A2I) and other regions in $\mathrm{PT}$ and $\mathrm{ST}$ ( $\mathrm{PT} \alpha, \mathrm{ST} \alpha$, and $\mathrm{STa}$ ) did not show such selectivity. A recent fMRI study has identified areas of T1b, T2, and T3 in the supratemporal plane (Scheich et al., 1998), which may correspond to $\mathrm{A} 1, \mathrm{~A} 2$, and $\mathrm{PT}$ in our study, respectively. The present study further revealed functional parcellation within A2, PT, and ST.

There are several possible differences between cognitive factors involved in the DIO condition and those in the DIC condition, which could produce the DICselective response pattern. First, an interaural stimuIus difference is present only under the DIC condition. Second, spatial attention to either ear is involved under the DIC condition. The subject has to direct attention to one ear when a target is presented and to switch attention to the other ear when a target is shifted to the other side. Third, selective attention, which is related to figure-background segregation, is required only under the DIC condition. The subject has to process an acoustically complex mixture of a target and a nontarget in order to extract a target from that mixture. F ourth, the DIC condition may require maintaining a higher state of alertness than the DIO condition because the DIC task might be more difficult, as indicated by its lower accuracy. However, we should note the absence of difference in RT as to behavioral control for task difficulty.

The rate of stimulus presentation may be another factor for the difference observed between the DIO and DIC conditions. According to previous PET studies (Price et al., 1992, 1996), the primary auditory cortex and middle regions of ST showed a linear relationship between their presentation rate and the blood flow response, while the activity in the left posterior ST (Wernicke's area) did not depend on the rate of presentation of heard words. Though the frequency of presenting meaningful phrases (target stimuli to which the subject attended) was equated for both conditions in our study, there was twice more acoustic material presented in the DIC condition relative to the DIO condition, which could possibly evoke DIC-selective responses in regions closely adjacent to Al. However, higher presentation rate of nonsense syllables (nontarget stimuli that the subject neglected) may not cause enhanced cortical activity under the DIC condition in regions that are distant from A1; PT, ST, and IF, for example. Recent fMRI studies reported that signal changes in the posterior periauditory regions exhibited a nonlinear (inverted $U$ ) relationship to word rate, reaching a peak at about 60 words per minute (wpm) (Büchel et al., 1998) or at $90 \mathrm{wpm}$ in the primary auditory cortex as well (Dhankhar et al., 1997). Furthermore, frontal regions showed a response to words irrespective of their presentation rate (Büchel et al., 1998).

It should be noted that the same response pattern in two regions may not reflect the same cognitive factors in each region. However, different response patterns in two regions reflect either the processing of different cognitive factors or a different sensitivity to the same factors. In either case, differential responses among multiple areas correspond to differences in cognitive function. Therefore, in the present study, we can conclude that the DIC-selective areas such as A2m, PT $\beta$, and ST $\beta$ are functionally distinct from other regions.

\section{Influence of Auditory Stimulation on the Activity of the Auditory Cortex}

Significant activity was observed in $\mathrm{A} 1$ and $\mathrm{A} 2$ under speech sound conditions (DIO and DIC), in comparison with the nonspeech sound condition (CON). This result indicates that these areas are related to the processing of complex temporal and spectral features of speech sounds at the early stages of auditory perception. Further studies are necessary to examine whether the activation in these areas reflects general spectro-temporal complexity or speech-specific acoustic characteristics instead. A recent fMRI study has reported voiceselective regions along the upper bank of the superior temporal sulcus (Belin et al., 2000), which may correspond to a higher stage of sound processing. It would be interesting to note the possibility that responses in voice-sel ective regions are enhanced by selective attention to human vocal sounds, as our results indicate that the response in ST $\beta$ is modulated by attentionrelated factors.

The masking effect of acoustic noise generated by scanner may influence responses in the auditory cortex, either by masking the auditory stimuli 
by the temporal overlaps with scanner noise (E dmister et al., 1999) or by saturating cortical responses to auditory stimuli in the presence of scanner noise (Talavage et al., 1999; Hall et al., 1999). As to the first point, we minimized acoustic contamination with scanner noise in the auditory stimuli (see Materials and Methods). For the second point, it has been reported that signal enhancement due to scanner noise was observed mainly in the primary auditory cortex (Bandettini et al., 1998; Talavage et al., 1999), whereas PT, ST, SMG, and IF were affected in less than half of the subjects tested (UImer et al., 1998). It is possible that, in the present experimental conditions, responses in the primary auditory cortex were affected by scanner noise. Nevertheless, consistent activation among subjects indicates absence of idiosyncratic effects of scanner noise in our experiments. On the other hand, it might be possible that an interaction with scanner noise made the DIC condition more difficult, which resulted in DIC-selective responses. However, we should note that task difference (DIC vs DIO) is the necessary condition to observe the differential responses, in which the physical characteristics of scanner noise were constant throughout each sequence.

\section{Functional Anatomy of Multiple Auditory Areas}

We found four regions around $\mathrm{HG}$ showing responses to speech sounds: $A 1, A 2 m, A 21$, and STa. With their close spatial relationship and their progressive increase in responses to both the DIO and DIC conditions, it is likely that these regions constitute a functional pathway in the order of A1, A2m, A2I, and STa. In other words, these regions have progressively greater selectivity for the stimuli presented. The auditory pathway proposed here corresponds to previous anatomical findings. Based on the gradient in acetylcholinesterase staining and cytochrome oxidase activity, a hierarchical order of A1, PA (posterior area), LA (lateral area), and STA (superior temporal area) has been proposed (Rivier and Clarke, 1997). A1 (TC of (von Economo and Horn, 1930)) was surrounded posteromedially by PA (the posterior part of TA) and posterolaterally by LA (TB) al ong HS. Moreover, STA (lateral part of TA) was on the STG, which locates lateral to LA. Except the other "intermediate level" auditory areas in that study, AA (anterior area) and MA (medial area), we found corresponding regions in our $\mathrm{fMRI}$ study. Specifically, the spatial configuration of $A 1, P A$, $L A$, and STA corresponds to that of $A 1, A 2 m, A 21$, and STa (Fig. 3). It is notable that the anatomical study and our functional study match with respect to the anatomical location of these areas and the order of the proposed hierarchical levels.

The auditory cortex of nonhuman primates has been extensively studied, and the concept of dividing the auditory cortex into core, belt, and parabelt areas has been proposed based on several different neuroanatomical techniques, including retrograde tracer injections and histochemical staining (Pandya and Sanides, 1973; Hackett et al., 1998). The core areas are located inside the lateral sulcus, laterally surrounded by the belt areas. The parabelt areas are further lateral to the belt and located on the exposed surface of the superior temporal gyrus. A hierarchical processing along core, belt, and parabelt has been suggested (Kaas et al., 1999). This hierarchical order has been partially confirmed by an electrophysiological study, showing that neurons in the belt area prefer certain complex stimuli, in contrast to neurons in the core area (Rauschecker et al., 1995). While Al corresponds to the core area (Pandya and Sanides, 1973), the belt area corresponds to A2, though a subdivision has been suggested (Hackett et al., 1998). It is possible that $A 2 m$ and $A 2 l$ of the present human study are related to the belt area, and that regions identified in PT and ST (PT $\beta, \mathrm{PT} \alpha, \mathrm{ST} \beta$, $\mathrm{ST} \alpha$, and $\mathrm{STa}$ ) are related to the belt and parabelt areas. However, we should note the presence of some critical differences in structure and function between the human and nonhuman primate auditory cortex that make it difficult to find counterparts of PT and ST, which are language-related areas, in nonhuman primates. F urthermore, it has been known in many cortical areas, such as visual areas, that functional mapping is able to divide an anatomically unseparated region into multiple subregions (Van Essen et al., 1998). Our functional parcellation in PT and ST indicates that future studies with some histochemical markers may clarify their anatomical substrates at the cellular level, just like A1, A2m, A2l, and STa.

\section{Functional Differentiation in the Frontal Lobe}

The result of the present study suggested that the inferior frontal area is also related to speech recognition as to the DIO and DIC conditions. This area was shown to differentiate into multiple regions, as well as in PT and ST. It is notable that both cortical language areas, Wernicke's area and Broca's area, are parcellated into two types of regions with different response patterns.

We observed DIC-selective response in the right anterior insula, and this region may correspond to AIA (anterior insula area) in a previous cytoarchitectual work (Rivier and Clarke, 1997). A functional imaging study (Griffiths et al., 1994) reported that the right anterior insula was selectively activated by apparent sound motion. The activation of this region in our study may reflect common factors between dichotic listening and auditory motion perception, such as spatial attention or interaural stimulus differences. The role of the anterior insula, however, needs to be further studied because we found a less selective region $(\alpha)$ in the right anterior insula of some subjects (Table 2). 


\section{Multiple Auditory Pathway Hypothesis}

Since a dichotic listening paradigm was established for the study of sel ective listening (Cherry, 1953), some models of selective attention have been proposed (Treisman, 1969; Posner and Dehaene, 1994; Sakai and Miyashita, 1994). In our paradigm, the DIC condition requires selective attention, which is related to figurebackground segregation. If auditory areas at the early stage process both the story phrases and the nonsense syllables in the course of figure-background segregation, the DIC condition would elicit larger responses than the DIO condition. In contrast, at the later stage, after filtering out the nonsense syllables as unattended stimuli, responses to the DIO and DIC conditions would become comparable. This "filter" process agrees with the decrease in DSI along the pathway of A2m, A2I, and STa.

Other than selective attention and acoustic differences, the interaural stimulus difference and spatial attention may also result in the DIC-selective responses of $A 2 \mathrm{~m}, \mathrm{PT} \beta, \mathrm{ST} \beta$, and IF $\beta$. On the other hand, cognitive factors such as speech sound recognition, which are independent of interaural difference, would be processed in areas with comparable responses to both conditions, that is, $\mathrm{PT} \alpha, \mathrm{ST} \alpha, \mathrm{SMG}$, and IF $\alpha$. It is possible that the pathway of $\mathrm{A} 2 \mathrm{~m}, \mathrm{PT} \beta, \mathrm{ST} \beta$, and IF $\beta$ is critical in orienting the location of sound sources by using dichotic cues, whereas the pathway of $\mathrm{PT} \alpha, \mathrm{ST} \alpha$, SMG, and IF $\alpha$ is useful for processing the patterns of speech sounds themselves. A recent study reported both inferior and superior parietal activation at the slices of $z>40$ during a spatial localization task (Bushara et al., 1999), while SMG activation in our study was found at lower slices $(z<32)$, suggesting further parcellation in the posterior parietal lobe. This dichotomy of the central auditory pathways is relevant to previously proposed "what" and "where" mechanisms in audition (Deutsch and Roll, 1976; Romanski et al., 1999), similar to the visual system in primates (Mishkin et al., 1983). Our findings further suggest that $\mathrm{A} 2$ and cortical language areas, such as Wernicke's and Broca's areas, play a pivotal role in combining these two mechanisms and in processing language beyond the primary auditory area.

\section{ACKNO WLEDGMENTS}

We thank Dr. J uro Kawachi for his encouragement, Dr. Albert Galaburda and Dr. David Embick for their helpful comments, Mr. Hiroki Sato and Mr. Tatsuya Takeuchi for their technical assistance, and Ms. Hiromi Matsuda for her administrative assistance. This research was supported by an ICORP grant from J ST to Y.M., and a CREST grant from J ST to K.L.S.

\section{REFERENCES}

Alho, K., Medvedev, S. V., Pakhomov, S. V., Roudas, M. S., Tervaniemi, M., Reinikainen, K., Zeffiro, T., and Näätänen, R. 1999. Se lective tuning of the left and right auditory cortices during spatially directed attention. Cognit. Brain Res. 7: 335-341.

Bandettini, P. A., J esmanowicz, A., Van Kylen, J ., Birn, R. M., and Hyde, J. S. 1998. Functional MRI of brain activation induced by scanner acoustic noise. Magn. Reson. Med. 39: 410-416.

Belin, P., Zatorre, R. J ., Lafaille, P., Ahad, P., and Pike, B. 2000. Voice-selective areas in human auditory cortex. Nature 403: 309312.

Brodmann, K. 1909. VergleichendeL okal isationslehreder Grosshirnrinde in ihren Prinzipien dargestelt auf Grund des Zellenbaues, Verlag von J ohann Ambrosius Barth, Leipzig.

Bushara, K. O., Weeks, R. A., Ishii, K., Catalan, M.-J., Tian, B., Rauschecker, J . P., and Hallett, M. 1999. Modality-specific frontal and parietal areas for auditory and visual spatial localization in humans. Nat. Neurosci. 2: 759-766.

Büchel, C., Holmes, A. P., Rees, G., and Friston, K. J . 1998. Characterizing stimulus-response functions using nonlinear regressors in parametric fMRI experiments. Neuroimage 8: 140-148.

Cherry, E. C. 1953. Some experiments on the recognition of speech, with one and with two ears. J. Acoust. Soc. Am. 25: 975-979.

Deutsch, D., and Roll, P. L. 1976. Separate "what" and "where" decision mechanisms in processing a dichotic tonal sequence. J . Exp. Psychol. (Hum. Percept. Perform.) 2: 23-29.

Dhankhar, A., Wexler, B. E., Fulbright, R. K., Halwes, T., Blamire, A. M., and Shulman, R. G. 1997. Functional magnetic resonance imaging assessment of the human brain auditory cortex response to increasing word presentation rates. J . Neurophysiol. 77: 476483.

Edmister, W. B., Talavage, T. M., Ledden, P. J ., and Weisskoff, R. M. 1999. I mproved auditory cortex imaging using clustered volume acquisitions. Hum. Brain Mapp. 7: 89-97.

Felleman, D. J ., and Van Essen, D. C. 1991. Distributed hierarchical processing in the primate cerebral cortex. Cereb. Cortex 1: 1- 47.

Frith, C. D., and Friston, K. J . 1996. The role of the thalamus in "top down" modulation of attention to sound. Neuroimage 4: 210-215.

Fujiwara, N., Nagamine, T., I mai, M., Tanaka, T., and Shibasaki, H. 1998. Role of the primary auditory cortex in auditory selective attention studied by whole-head neuromagnetometer. Cognit. Brain Res. 7: 99-109.

Galaburda, A. M., and Sanides, F. 1980. Cytoarchitectonic organization of the human auditory cortex. J . Comp. Neurol. 190: 597-610.

Geschwind, N. 1979. Specializations of the human brain. Sci. Am. 241: 158-168.

Grady, C. L., Van Meter, J . W., Maisog, J . M., Pietrini, P., Krasuski, J ., and Rauschecker, J. P. 1997. Attention-related modulation of activity in primary and secondary auditory cortex. NeuroReport 8: 2511-2516.

Griffiths, T. D., Bench, C. J ., and Frackowiak, R. S. J . 1994. Human cortical areas selectively activated by apparent sound movement. Curr. Biol. 4: 892-895.

Hackett, T. A., Stepniewska, I., and Kaas, J . H. 1998. Subdivisions of auditory cortex and ipsilateral cortical connections of the parabelt auditory cortex in macaque monkeys. J . Comp. Neurol. 394: 475495.

Hall, D. A., Haggard, M. P., Akeroyd, M. A., Palmer, A. R., Summerfield, A. Q., Elliott, M. R., Gurney, E. M., and Bowtell, R. W. 1999. "Sparse" temporal sampling in auditory fMRI. Hum. Brain Mapp. 7: 213-223.

Hashimoto, R., Homae, F., Nakajima, K., Miyashita, Y., and Sakai, K. 1999. Attentional influence on speech recognition: An fMRI study of multiple auditory areas. Neuroimage 9: S1057.

Hutsler, J . J ., and Gazzaniga, M. S. 1996. Acetylcholinesterase staining in human auditory and language cortices: Regional variation of structural features. Cereb. Cortex 6: 260-270. 
J äncke, L., Mirzazade, S., and Shah, N.J . 1999. Attention modulates activity in the primary and the secondary auditory cortex: A functional magnetic resonance imaging study in human subjects. Neurosci. Lett. 266: 125-128.

Kaas, J . H., Hackett, T. A., and Tramo, M. J . 1999. Auditory processing in primate cerebral cortex. Curr. Opin. Neurobiol. 9: 164-170.

Kim, K. H. S., Relkin, N. R., Lee, K. M., and Hirsch, J . 1997. Distinct cortical areas associated with native and second languages. Nature 388: $171-174$.

Mishkin, M., Ungerleider, L. G., and Macko, K. A. 1983. Object vision and spatial vision: Two cortical pathways. Trends Neurosci. 6: 414- 417.

Moffat, S. D., Hampson, E., and Lee, D. H. 1998. Morphology of the planum temporale and corpus callosum in left handers with evidence of left and right hemisphere speech representation. Brain 121: 2369-2379.

O'Leary, D. S., Andreasen, N. C., Hurtig, R. R., Hichwa, R. D., Watkins, G. L., Ponto, L. L. B., Rogers, M., and Kirchner, P. T. 1996. A positron emission tomography study of binaurally and dichotically presented stimuli: Effects of level of language and directed attention. Brain Lang. 53: 20-39.

Oldfield, R. C. 1971. The assessment and analysis of handedness: The Edinburgh inventory. Neuropsychologia 9: 97-113.

Pandya, D. N., and Sanides, F. 1973. Architectonic parcellation of the temporal operculum in rhesus monkey and its projection pattern. Z. Anat. Entwickl.-Gesch. 139: 127-161.

Penhune, V. B., Zatorre, R. J ., MacDonald, J . D., and Evans, A. C. 1996. Interhemispheric anatomical differences in human primary auditory cortex: Probabilistic mapping and volume measurement from magnetic resonance scans. Cereb. Cortex 6: 661- 672.

Posner, M. I., and Dehaene, S. 1994. Attentional networks. Trends Neurosci. 17: 75-79.

Price, C., Wise, R. J. S., Ramsay, S., Friston, K. J ., Howard, D., Patterson, K., and Frackowiak, R. S. J . 1992. Regional response differences within the human auditory cortex when listening to words. Neurosci. Lett. 146: 179-182.

Price, C. J ., Moore, C. J ., and Frackowiak, R. S. J . 1996. The effect of varying stimulus rate and duration on brain activity during reading. Neuroimage 3: 40-52.

Pugh, K. R., Shaywitz, B. A., Shaywitz, S. E., Fulbright, R. K., Byrd, D., Skudlarski, P., Shankweiler, D. P., Katz, L., Constable, R. T., Fletcher, J ., Lacadie, C., Marchione, K., and Gore, J. C. 1996. Auditory selective attention: An fMRI investigation. Neuroimage 4: 159-173.

Rademacher, J ., Caviness, V. S., J r., Steinmetz, H., and Galaburda, A. M. 1993. Topographical variation of the human primary cortices: Implications for neuroimaging, brain mapping, and neurobiology. Cereb. Cortex 3: 313-329.

Rauschecker, J. P., Tian, B., and Hauser, M. 1995. Processing of complex sounds in the macaque nonprimary auditory cortex. Science 268: 111-114.

Rivier, F., and Clarke, S. 1997. Cytochrome oxidase, acetylcholinesterase, and NADPH-diaphorase staining in human supratemporal and insular cortex: Evidence for multiple auditory areas. Neuroimage 6: 288-304.

Romanski, L. M., Tian, B., Fritz, J., Mishkin, M., Goldman-Rakic, P. S., and Rauschecker, J. P. 1999. Dual streams of auditory afferents target multiple domains in the primate prefrontal cortex. Nat. Neurosci. 2: 1131-1136.

Sakai, K., and Miyashita, Y. 1994. Visual imagery: An interaction between memory retrieval and focal attention. Trends Neurosci. 17: 287-289.
Sakai, K., Watanabe, E., Onodera, Y., I tagaki, H., Yamamoto, E., Koizumi, H., and Miyashita, Y. 1995a. Functional mapping of the human somatosensory cortex with echo-planar magnetic resonance imaging. Magn. Reson. Med. 33: 736-743.

Sakai, K., Watanabe, E., Onodera, Y., Uchida, I., Kato, H., Yamamoto, E., Koizumi, H., and Miyashita, Y. 1995b. Functional mapping of the human colour centre with echo-planar magnetic resonance imaging. Proc. R. Soc. Lond. Biol. 261: 89-98.

Scheich, H., Baumgart, F., Gaschler-Markefski, B., Tegeler, C., Tempelmann, C., Heinze, H. J ., Schindler, F., and Stiller, D. 1998. Functional magnetic resonance imaging of a human auditory cortex area involved in foreground-background decomposition. Euro. J . Neurosci. 10: 803-809.

Stehling, M. K., Turner, R., and Mansfield, P. 1991. Echo-planar imaging: Magnetic resonance imaging in a fraction of a second. Science 254: 43-50.

Steinmetz, H. 1996. Structure, function and cerebral asymmetry: In vivo morphometry of the planum temporale. Neurosci. Biobehav. Rev. 20: 587-591.

Steinmetz, H., Rademacher, J., Huang, Y., Hefter, H., Zilles, K., Thron, A., and Freund, H.-J. 1989. Cerebral asymmetry: MR planimetry of the human planum temporale. J . Comput. Assist. Tomogr. 13: 996-1005.

Talairach, J ., and Tournoux, P. 1988. Co-Planar Stereotaxic Atlas of the Human Brain. 3-Dimensional Proportional System: An Approach to Cerebral I maging, Thieme, Stuttgart.

Talavage, T. M., Edmister, W. B., Ledden, P. J ., and Weisskoff, R. M. 1999. Quantitative assessment of auditory cortex responses induced by imager acoustic noise. Hum. Brain Mapp. 7: 79-88.

Treisman, A. M. 1969. Strategies and models of selective attention. Psychol. Rev. 76: 282-299.

Tzourio, N., Massioui, F. E., Crivello, F., J oliot, M., RenaulT, B., and Mazoyer, B. 1997. Functional anatomy of human auditory attention studied with PET. Neuroimage 5: 63-77.

Ulmer, J . L., Biswal, B. B., Yetkin, F. Z., Mark, L. P., Mathews, V. P., Prost, R. W., Estkowski, L. D., McAuliffe, T. L., Haughton, V. M., and Daniels, D. L. 1998. Cortical activation response to acoustic echo planar scanner noise. J . Comput. Assist. Tomogr. 22: 111119.

Van Essen, D. C., Drury, H. A., J oshi, S., and Miller, M. I. 1998. Functional and structural mapping of human cerebral cortex: Solutions are in the surfaces. Proc. Natl. Acad. Sci. USA 95: 788795.

von Economo, C., and Horn, L. 1930. Über Windungsrelief, Maße und Rindenarchitektonik der Supratemporalfläche, ihre individuellen und ihre Seitenunterschiede. Z. Neurol. Psychiat. 130: 678757.

Westbury, C. F., Zatorre, R. J ., and Evans, A. C. 1999. Quantifying variability in the planum temporale: A probability map. Cereb. Cortex 9: 392-405.

Woldorff, M. G., Gallen, C. C., Hampson, S. A., Hillyard, S. A., Pantev, C., Sobel, D., and Bloom, F. E. 1993. Modulation of early sensory processing in human auditory cortex during auditory selective attention. Proc. Natl. Acad. Sci. USA 90: 8722- 8726.

Zatorre, R. J ., Evans, A. C., Meyer, E., and Gjedde, A. 1992. Lateralization of phonetic and pitch discrimination in speech processing. Science 256: 846-849.

Zatorre, R. J., Mondor, T. A., and Evans, A. C. 1999. Auditory attention to space and frequency activates similar cerebral systems. Neuroimage 10: 544-554. 